Documentation et bibliothèques

DOCUMENTATION BIBLIOTHEQUES

\title{
Bibliothèques publiques québécoises et coopération régionale
}

\section{Jacques Panneton}

Volume 23, numéro 3, septembre 1977

URI : https://id.erudit.org/iderudit/1055225ar

DOI : https://doi.org/10.7202/1055225ar

Aller au sommaire du numéro

\section{Éditeur(s)}

Association pour l'avancement des sciences et des techniques de la documentation (ASTED)

\section{ISSN}

0315-2340 (imprimé)

2291-8949 (numérique)

Découvrir la revue

Citer cet article

Panneton, J. (1977). Bibliothèques publiques québécoises et coopération régionale. Documentation et bibliothèques, 23(3), 127-133.

https://doi.org/10.7202/1055225ar

\section{Résumé de l'article}

Les bibliothèques centrales de prêt et les bibliothèques municipales du Québec se sont développées en parallèle, sans effort systématique de coordination. À partir d'un rappel des notions de bibliothèque publique et de réseau, l'auteur identifie les limites et contingences du développement des bibliothèques centrales de prêt et municipales. Il insiste particulièrement sur la spécificité des besoins à combler par opposition aux critères basés sur la taille des municipalités pour décider de leur appartenance structurelle. Il propose ensuite un modèle souple de réseau régional de coordination qui fait appel au concept d'agglomération urbaine et tente d'intégrer les contingences identifiées.
Tous droits réservés ( $)$ Association pour l'avancement des sciences et des techniques de la documentation (ASTED), 1977
Ce document est protégé par la loi sur le droit d'auteur. L'utilisation des services d'Érudit (y compris la reproduction) est assujettie à sa politique d'utilisation que vous pouvez consulter en ligne.

https://apropos.erudit.org/fr/usagers/politique-dutilisation/ 


\title{
Bibliothèques publiques québécoises et coopération régionale
}

\author{
Jacques Panneton \\ Conservateur \\ Bibliothèque de la Ville de Montréal
}

Les bibliothèques centrales de prêt et les bibliothèques municipales du Québec se sont développées en parallèle, sans effort systématique de coordination. À partir d'un rappel des notions de bibliothèque publique et de réseau, l'auteur identifie les limites et contingences du développement des bibliothèques centrales de prêt et municipales. II insiste particulièrement sur la spécificité des besoins à combler par opposition aux critères basés sur la taille des municipalités pour décider de leur appartenance structurelle. II propose ensuite un modèle souple de réseau régional de coordination qui fait appel au concept d'agglomération urbaine et tente d'intégrer les contingences identifiées.

The bibliothèques centrales de prêt and the municipal libraries of Québec have developed simultaneously on parallel roads without any effort at systematic coordination. Starting from a reminder of the definitions of bibliothèque centrale de prêt and municipal libraries, the author identifies the limits and conditions of the development of these libraries. He insists on the specific nature of the needs to be filled, rather than on criteria based on the size of municipality, to decide on the type of library structure needed. He, then, proposes a model of a flexible coordinated regional network which draws upon the concept of urban communities and attempts to integrate the different contingencies identified.

Las bibliotecas de préstamo central y las bibliotecas municipales de Quebec se han desarrollado en paralelo sin esfuerzo de coordinación sistemática. Habiendo recordado las nociones de bibliotecas de préstamo central y de red, el autor identifica los limites y las contingencias del desarrollo de las bibliotecas de préstamo central y municipales. Insiste particularmente en la especificidad de las necesidades que hay que colmar en oposición con los criterios que se basan en la dimensión de las municipalidades para colocar cada biblioteca en su estructura respectiva. Después propone un modelo flexible de red regional de coordinación que recurre al concepto de aglomeración urbana e intenta una integración de las contingencias identificadas.

Le problème de la coopération régionale entre les bibliothèques publiques québécoises, entre les bibliothèques municipales et les bibliothèques centrales de prêt nous donne le goût d'un apologue.

Un confrère illustre a déjà comparé nos bibliothèques centrales de prêt à Cendrillon (ou était-ce aú Petit Poucet?): cela fait un bien piètre personnage de conte moral, outre qu'il n'ait pas alors précisé si la pantouffle était de verre ou de vair. Les signes de richesse sont d'ailleurs très relatifs. La ventripotence manifeste autant le rachitisme que l'obésité lorsque la perspective s'élargit au-delà du nombril. 
Quant à nos bibliothèques municipales, apparaissant plus proches de la bibliothèque publique de droit, nous leur trouvons des allures de Rosines. De Rosines rossinantes pour dire vrai, ce qui ne vaut guère mieux. Nous avons beau alors essayer toutes les inversions, transpositions et substitutions, entortiller Cervantes, Beaumarchais, Mozart et Rossini, nous n'arrivons pas à filer une allégorie subtile et civilisée avec pareil fuseau (à développer ultérieurement: les rapports entre la bibliothéconomie québécoise et la Belle au bois dormant).

II reste l'approche inimaginative, terne, indolente et indolore, paresseuse mais vaniteuse, manieuse de citations et de références, l'approche technocratique, donc subjective et autogratifiante.

\section{Trois concepts}

Disposons comme une grille trois notions auxquelles nous ferons référence en discutant plus loin de coopération et de coordination.

Premièrement, la bibliothèque publique. Le Comité conjoint ABQ-ACBLF sur la législation des bibliothèques publiques du Québec en a produit une définition qui reprend tous les éléments qui se sont historiquement greffés à la notion de départ:

"La bibliothèque publique est une institution qui rassemble, de façon impartiale la documentation, sous quelque forme que ce soit, que nécessite la vie des individus et des groupes. Elle ordonne cette documentation, la rend facile d'accès et joue un rôle actif dans sa diffusion et son utilisation. Elle est gratuite et ouverte à tous indistinctement.»

Nous insistons sur l'élément «joue un rôle actif dans sa diffusion et son utilisation», car il inscrit dans la définition le caractère essentiel de la relation d'aide entre la bibliothèque et son usager, celle qui fonde les accessibilités intellectuelle et psychologique; cet élément distingue la bibliothèque du dépôt de livre maquillé.
Deuxièmement, le réseau. Citons à nouveau le Comité conjoint:
"Un ensemble de bibliothèques publi- ques unies par des liens formels et dont les ressources et les services sont mis à la disposition des bi- bliothèques constituant cet ensemble, ou encore des citoyens habitant le territoire desservi par ce groupe de bibliothèques ou les deux à la fois."

Cette définition implique un ensemble, une organisation formelle indéfinie mais stable, un système de communication et des opérations multidirectionnelles.

Dans une étude récente sur le rôle et le financement des bibliothèques centrales de prêt, et qui pourrait avoir des répercussions importantes, Jean Ouellet, à partir du concept de réseau, élabore:

"L'organisation régionale pour sa part est fondée sur deux principaux éléments: une bibliothèque ressource; un organisme régional de coordination qui s'occupe du service aux bibliothèques locales. Dépendant beaucoup des éléments qui déjà sont en place et de la situation qui prévaut au niveau du potentiel, différents modèles sont en vigueur dont voici à notre avis l'alternative de base: lorsque au niveau local des bibliothèques peuvent être identifiées comme étant ou pouvant être des points forts, il est possible alors que l'organisme régional soit, totalement ou en partie, fondé sur ces bibliothèques ressources, noyau du système; inversement, lorsque au niveau local des points forts n'existent pas et lorsqu'on ne prévoit pas qu'il puisse en exister, la situation contraire est de mise et le centre de ressources, noyau du système à former, peut alors être associé à l'organisme régional luimême.»

Nous le citons pour faire ressortir qu'il s'exclut de la notion de réseau lorsqu'il fait équivaloir dans le deuxième terme de l'al- 
ternative, la bibliothèque ressource et l'organisation de coordination. II n'y a plus réseau mais bibliothèque unique à cause d'une confusion entre objectifs et stratégie.

La notion de réseau est, par ailleurs, totalement absente des règlements de subvention aux bibliothèques publiques de la Commission des bibliothèques publiques du Québec.

Troisièmement, ce que nous appellerons l'irradiation de l'autonomie municipale. C'est chercher à voir ici aux rayons $X$ le réflexe politique des petites municipalités placées devant une offre de service. Face à l'opportunité d'établir un service de bibliothèque, la première (et seule) question concerne le coût. Si une alternative existe entre un service de qualité satisfaisante à coût supérieur et un service primaire à moindre coût, ce second terme est automatiquement choisi, surtout s'il s'accompagne d'une disparition de responsabilité administrative directe. Ce réflexe est à la base du succès rapide des bibliothèques centrales de prêt. Ce qui revient à dire que la Commission des bibliothèques publiques du Québec doit fixer en vue de quels objectifs de bibliothèques et dans quel encadrement devra s'exercer la liberté (autonomie) municipale, dans ce secteur d'activité. $\mathrm{Ne}$ pas admettre cette nécessité, c'est refuser de constater que, dans ce cas, l'autonomie n'habille le plus souvent d'autre squelette que le vouloir-minimum (qui engendre malheureusement le salaire-suffisant).

\section{La situation}

On a trop écrit que la Loi des bibliothèques publiques du Québec est jeune (1959) et qu'elle permet de tout faire dans le respect de l'autonomie municipale. Après tout, elle est majeure (18 ans) et elle a plutôt permis de ne rien faire d'organisé (lire: de faire du non-organisé).

Nous avons donc développé deux sortes de bibliothèques publiques, des municipales et des centrales de prêt. Elles sont si mal et si obscurément coordonnées qu'on a pu voir un ministre des Affaires culturelles proposer qu'elles relèvent de deux services différents du ministère.

Les bibliothèques centrales de prêt sont puissantes par ambiguïté. Elles (celles qui ont été créées) se sont développées rapidement et ont manifestement comblé un besoin. Une constatation explique partiellement ce succès: elles sont apparues dans des secteurs du Québec où était absente toute structure culturelle locale de base, en dehors des comités locaux qu'elles créaient elles-mêmes. Jean Ouellet fait ressortir en effet que, dans les municipalités dont la population est inférieure à 5,000 habitants (celles que doivent desservir les bibliothèques centrales de prêt), les recoupements avec d'autres structures d'intervention culturelle globale ne toucheraient que $12 \%$ de la population visée. Les bibliothèques centrales de prêt ont alors été considérées comme le seul moyen d'intervention culturelle globale. Elles ont de fait occupé ce champ. Ce qui leur a d'ailleurs valu une image dynamique et un caractère d'évidente nécessité.

Mais elles ne sont pas (uniquement du moins) des moyens d'intervention culturelle globale. Elles sont moyen d'intervention sectorielle, elles sont bibliothèques. Précisons tout de suite que nous ne désirons pas discuter ici du mérite ou du démérite de leur intervention globale, ni du rôle plus ou moins large qu'elles devraient jouer dans ce secteur. La distinction capitale étant faite, l'ambiguité favorable étant levée, elles ne nous intéressent plus qu'en tant que bibliothèques, bien que sectoriellement elles soient sensiblement moins intéressantes.

Comme bibliothèques publiques elles sont en effet très lourdement déficientes. Nous avons déjà écrit que les bibliothèques centrales de prêt représentent une étape nécessaire mais non terminale. Entendons par là qu'elles ont solutionné, sectoriellement, un seul problème, celui de l'accessibilité physique, ce qu'il fallait faire. Elles sont donc étapes, non solution. Elles doi- 
vent être encadrées, ne doivent pas englober. Du point de vue de la qualité des services (voir le premier concept) elles font consortium de bibliothèques paroissiales. Leurs carences en locaux, personnel de service (quantité et qualité), heures d'ouverture, spécificité des collections, niveau de service, sont très importantes.

Elles ne peuvent d'ailleurs faire mieux à cause de l'isolement (souvent volontaire) où elles se tiennent et surtout de leur configuration. L'accroissement du niveau de service de l'ensemble de microbibliothèques qu'elles constituent ne peut être acquis autrement que par l'articulation avec des bibliothèques ressources au niveau de chaque région, un minimum de concentration des ressources étant nécessaire à l'élévation du niveau de complexité. Or elles ne sont pas ces bibliothèques ressources: en tant que bibliothèques, elles n'existent pas centralement, elles constituent une juxtaposition de petites collections relativement faibles, ausși rotatives soient-elles.

L'autre volet du problème est que les bibliothèques ressources régionales n'existent pas nécessairement. En effet, toutes les régions ne sont pas dotées d'agglomérations urbaines suffisamment développées pour permettre la constitution de bibliothèques d'une taille suffisante. De plus, les bibliothèques municipales existantes sont très en deçà des normes du Service des bibliothèques publiques du Québec à tous points de vue. Elles ont en commun cependant une parenté quant à la nature de leurs problèmes de service, parenté qu'elles n'ont pas avec les bibliothèques centrales de prêt.

Comme l'ensemble des bibliothèques publiques québécoises n'a pas été dessiné, mais s'est constitué empiriquement, nous sommes ici confrontés à la normalisation de ce lien de parenté. Des bibliothèques municipales autonomes existent qui desservent moins de 5,000 âmes, des bibliothèques centrales de prêt existent qui doivent desservir les municipalités de 5,000 âmes et moins, mais qui desservent audelà, la moitié seulement des municipalités de plus de 5,000 âmes sont dotées d'une bibliothèque autonome et, dans ce groupe, le tiers seulement des municipalités dont la population se situe entre 5,000 et 10,000 habitants. Implantez alors des bibliothèques centrales de prêt partout, superposez toutes ces réalités dans chaque région et essayez d'articuler ces ensembles.

L'étude économique de Jean Ouellet révèle que les municipalités les moins capables financièrement de supporter une bibliothèque publique qui réponde aux normes québécoises sont les municipalités de 5,000 à 10,000 âmes:

"Les petites villes sont dans leur ensemble moins riches et il est clair que le coût de la bibliothèque qui leur est imposé est lourd: le taux d'imposition représentant cette dépense est en effet de $\$ 0.45$ du $\$ 100$ d'évaluation, par rapport à un ...coût de $\$ 0.25 \ldots$ du $\$ 100$ dans le cas des villes de 5,000 à 10,000 habitants... Ceci vient confirmer dans un sens le bien fondé d'une aide particulière à apporter aux petites villes inférieures à 5,000 habitants... Enfin, si on considère la capacité financière des villes non seulement à partir du critère de la richesse foncière, mais aussi en tenant compte du niveau de dépenses, les calculs montrent, lorsque les villes sont prises globalement par taille, que ce seraient alors les villes ayant entre 5,000 et 10,000 habitants qui auraient le plus de difficultés...»

Ouellet proposera plus loin que les bibliothèques centrales de prêt desservent les municipalités de moins de 10,000 âmes. Cette suggestion est basée sur le faible coefficient de recoupement avec d'autres structures d'intervention globale et sur la difficulté de payer pour ces municipalités.

Ce n'est pas une solution convenable: premièrement le motif d'intervention globale n'est pas pertinent au secteur des bibliothèques et, deuxièmement, la non-capacité de payer n'engendre pas de parenté en termes de nature des services comman- 
dés. Le seul résultat concret est la preuve économique d'un certain seuil de viabilité pour la bibliothèque autonome. LowellMartin avait déjà proposé 50,000 âmes, mais des exemples québécois tendent à montrer la possibilité d'un seuil plus faible chez nous. On peut fort bien concevoir en effet qu'une municipalité commande un service de caractère urbain avec une faible population (toutes les banlieues immédiates des villes de moyenne importance) alors qu'une autre municipalité de plus forte taille affiche ouvertement des structures paroissiales (Bécancour). II faut définitivement abandonner le concept de populationcharnière comme devant définir l'appartenance à un type de structure et lui substituer celui de municipalité assistable, qu'on pourrait provisoirement situer à 10,000 âmes, l'assistance pertinente étant considérée en fonction de la nature et non de la taille de la municipalité. Encore faut-il que cette assistance soit perçue comme visant à l'utilisation maximale des ressources et non à la réalisation d'économies; aucun recul n'est concevable par rapport aux normes du Service des bibliothèques publiques du Québec, peu importe le schéma d'aménagement qui sera choisi.

\section{Une solution}

À partir de la nécessité de provoquer une accélération du développement des bibliothèques publiques, de la nécessité économique de prêter assistance aux municipalités de moins de 10,000 âmes, de la nécessité de respecter en termes de spécificité de services la nature des agglomérations à desservir, de l'incapacité des bibliothèques centrales de prêt d'évoluer au-delà de leur actuel niveau de complexité, est-il possible d'établir un mode de coopération et de coordination régionale qui permette à la fois une maximisation de l'usage des ressources actuelles et une incitation au développement? Conceptuellement, il existe une solution de type réglementaire.

La Commission et le Service des bibliothèques publiques du Québec doivent se doter d'un plan de développement des bibliothèques publiques québécoises qui soit autre chose qu'un catalogue d'opportunités. Les règlements de subvention doivent devenir l'articulation d'une implantation de caractère spécifique: ils canaliseront, par des mesures généralement incitatives, parfois coercitives, un type d'intervention municipale à privilégier. De sorte que chaque municipalité sera libre dans ses décisions d'établir et de gérer une bibliothèque, mais le droit aux subventions sera acheté par l'observance d'un certain nombre de règles précises. Nous tentons ici d'intégrer dans une solution possible les contraintes indiquées plus haut.

II faut introduire dans le plan et les règlements la notion de réseau. Cette notion permettra la mise sur pied de structures régionales de coopération dont il est préférable de ne pas tracer de modèle univoque. Rappelons qu'un réseau n'est pas assimilable, jamais, à une bibliothèque principale (ou ressource). Le réseau n'est cependant pas concevable sans bibliothèque(s) ressource(s).

La notion théorique nécessaire au développement naturel d'une bibliothèque ressource est l'agglomération urbaine. Les travaux de l'Office de planification et de développement du Québec (O.P.D.Q.) peuvent ici être mis à profit. L'existence d'une agglomération suffisante permet la concentration des ressources nécessaire au développement qualitatif des services. Selon les circonstances et au gré des autorités locales, l'agglomération pourrait être desservie par une bibliothèque unique ou par des bibliothèques autonomes coordonnées régionalement. Toutes les municipalités des agglomérations désignées seraient desservies par une bibliothèque de type urbain, sans égard à leur taille. Les règlements de subvention visent dans ce cas à forcer plus ou moins à la. coopération, non à la fusion.

Les municipalités situées à l'extérieur des agglomérations désignées sont desservies par une bibliothèque autonome affiliée au réseau si la population dépasse 10,000 âmes. Lorsque la population est inférieure à 10,000 âmes, la municipalité est desser- 
vie par la bibliothèque centrale de prêt si elle présente des caractéristiques socioéconomiques de type rural, ou par une bibliothèque autonome appuyée fortement par le réseau si elle présente des caractéristiques urbaines.

La structure régionale de coordination n'est jamais une bibliothèque particulière. Ceci n'est pas opportun politiquement: plusieurs expériences récentes font ressortir la nécessité de laisser les autorités locales négocier et dessiner leurs modes de regroupement. Ce n'est pas recevable administrativement: il répugne de confier un pouvoir de coordination sur un ensemble territorial à une institution dont la nature et le rôle sont non spécifiques par rapport aux problèmes posés. Ce principe reste vrai, que l'institution tentativement coordonnatrice soit bibliothèque centrale de prêt ou municipale autonome ressource. Le réseau régional est plutôt le regroupement des bibliothèques publiques de la région constituées en réseau subventionné comme tel: bibliothèque d'agglomération, bibliothèques autonomes, bibliothèque centrale de prêt. Selon qu'elles sont unifiées ou autonomes, les bibliothèques des agglomérations désignées sont coordonnées par la bibliothèque d'agglomération ou par le réseau régional.

Selon la configuration particulière à chaque région et selon le degré d'intégration des services de bibliothèque au niveau des agglomérations désignées, le partage des responsabilités de programmes entre le réseau régional et les bibliothèques d'agglomération peut varier. Les principaux champs de coopération à explorer sont les suivants:

- réciprocité des services

- catalogue collectif des ressources

- prêt entre bibliothèques

- services de référence

- collections rotatives de livres, disques, diapositives, etc.

- information régionale
- coordination avec les bibliothèques d'enseignement

- entreposage commun de la documentation spécialisée d'usage limité

- centralisation régionale des services techniques

- publicité coordonnée.

Le lecteur aura sans doute remarqué que nous ne sommes plus très loin des recommandations du Comité conjoint cité plus haut.

Stratégiquement, cette question doit être ouvertement débattue au sein de nos associations dans les suites et non-suites des initiatives pertinentes aux problèmes de coopération. II ne suffit pas en effet qu'une solution, celle-ci ou une autre, soit développée avec plus ou moins de précision pour qu'elle se trouve implantée. Quelqu'un doit s'en occuper. Par exemple, l'Étude Aubry-Denis sur les bibliothèques publiques de la région de Montréal a été réalisée pour le ministère des Affaires culturelles et contient un ensemble de recommandations qui concernent souvent d'autres niveaux d'autorité que les bibliothèques concernées. Ce sont elles néanmoins qui ont pris l'initiative formelle: la décision administrative importe plus que le cheminement suivi.

D'autre part, un excellent moyen d'obtenir une prise de position quelconque sur ces problèmes de la part du Gouvernement du Québec serait peut-être qu'une région soumette à la Commission des bibliothèques publiques du Québec un projet spécifique.

Nous avons délibérément omis de traiter des problèmes de coopération entre les bibliothèques publiques et les autres systèmes d'information. Nous n'ignorons pas, ni ne contestons leur importance. Un étapisme en valant bien un autre, il semble toutefois plus important, présentement, de mettre de l'ordre dans l'arrière-cour. Nos propres problèmes de coopération sont déjà suffisamment compliqués à détortiller. 
Même en termes exclusifs de bibliothèques, plusieurs problèmes ne sont pas résolus. En voici quelques-uns:

- modèle administratif convenant le mieux aux conditions politiques et bibliothéconomiques des régions;

- modèle de partage des interventions entre bibliothèques d'agglomération, bibliothèques autonomes et bibliothèques centrales de prêt;

- mode de financement des programmes de coopération, des bibliothèques d'agglomération et des réseaux régionaux;

- mode de contrôle gouvernemental en regard des objectifs des régions;

- critères pour le choix de la source du soutien aux bibliothèques autonomes situées à l'extérieur des agglomérations.

Réal Messier écrivait en 1973:

"ll est donc souhaitable que de nouveaux règlements soient formulés, règlements plus incitatifs et même coercitifs, où l'on insisterait pour que l'effort financier soit de caractère municipal et qu'on n'attende pas que le gouvernement assume l'entière responsabilité. Trop souvent, les municipalités n'ont fourni que le montant minimum pour avoir droit à la subvention et se sont contentées de ces sommes pour offrir un service de bibliothèque publique.»

À notre avis, le raisonnement s'applique mutatis mutandis à la coopération, qui est également affaire de pouvoirs locaux, dont les directeurs de bibliothèques.

L'objectif essentiel que nous avons visé a été la présentation d'un modèle viable qui évite les erreurs prévisibles dans la définition du contenant et qui laisse le soin du contenu aux bibliothèques et aux régions concernées. Nous soumettons, à notre avis, un modèle souple, et dans la mesure même de cette souplesse, les hypothèses exclues le sont catégoriquement.

\section{Bibliographie}

Agglomérations urbaines du Québec; essais de détermination et de délimitation. Québec, Direction générale de l'Office de planification et de développement du Québec, 1975.

Bowron, Albert. The Ontario Public Library; Review and Reorganisation. Toronto, Information, Media, and Library Planners, 1976. 184 p.

Castonguay, André et al. Rapport du Comité conjoint $A B Q-Q L A / A C B L F$ sur la législation des bibliothèques publiques du Québec. Montréal, 1972. $63 \mathrm{p}$

Humphy, John A. "The place of urban main libraries in larger library networks", Library Trends, vol. 20, no. 4 (April 1972), 673-692.

Messier, Réal. "Les bibliothèques publiques au Danemark", Documentation et bibliothèques, vol. 19, no 4 (décembre 1973), 155-159.

Ouellet, Jean. Le rôle et le financement des bibliothèques centrales de prêt. Québec, Ministère des Affaires culturelles, non publié.

Québec (province), Ministère des Affaires culturelles, Commission des bibliothèques publiques. Règlement concernant l'attribution et le calcul des subventions. Québec, 1975. 9 p.

Québec (province), Ministère des Affaires culturelles, Service des bibliothèques publiques. Normes pour les bibliothèques municipales. Québec, 1974. $62 \mathrm{p}$. 